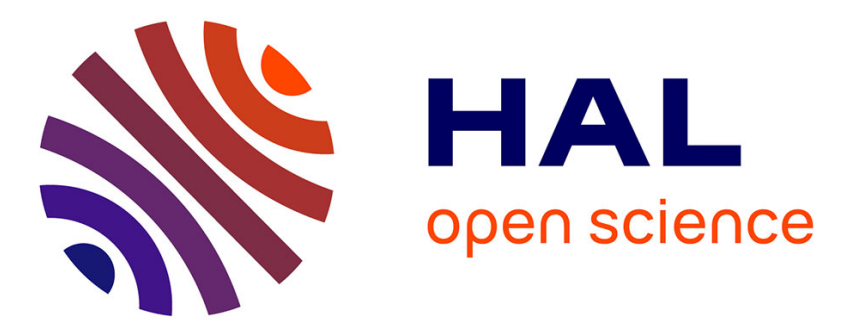

\title{
The bap module: A multisignal Integrator rrchestrating growth
}

\author{
Nathalie Boure, S. Vinod Kumar, Nicolas Arnaud
}

\section{To cite this version:}

Nathalie Boure, S. Vinod Kumar, Nicolas Arnaud. The bap module: A multisignal Integrator rrchestrating growth. Trends in Plant Science, 2019, 24 (7), pp.602-610. 10.1016/j.tplants.2019.04.002 . hal-02629211

\section{HAL Id: hal-02629211 \\ https://hal.inrae.fr/hal-02629211}

Submitted on 25 Oct 2021

HAL is a multi-disciplinary open access archive for the deposit and dissemination of scientific research documents, whether they are published or not. The documents may come from teaching and research institutions in France or abroad, or from public or private research centers.
L'archive ouverte pluridisciplinaire HAL, est destinée au dépôt et à la diffusion de documents scientifiques de niveau recherche, publiés ou non, émanant des établissements d'enseignement et de recherche français ou étrangers, des laboratoires publics ou privés.

\section{다)(1) $(5$}

Distributed under a Creative Commons Attribution - NonCommerciall 4.0 International 


\section{The BAP module: A multi-signal integrator orchestrating growth}

2

3 Nathalie Bouré ${ }^{1,2}$, S. Vinod Kumar ${ }^{3}$ and Nicolas Arnaud ${ }^{1 *}$

4 Affiliations:

5

6 Versailles, France

${ }^{2}$ Univ. Paris-Sud, Université Paris-Saclay, 91405 Orsay, France *Correspondence: nicolas.arnaud@inra.fr (N. Arnaud). integration, growth control.

\section{Abstract} insights into the molecular control of growth.

\section{Coordination of growth: Enter the BAP module}

${ }^{1}$ Institut Jean-Pierre Bourgin, INRA, AgroParisTech, CNRS, Université Paris-Saclay, 78000

${ }^{3}$ Department of Biosciences, University of Exeter, Stocker Road, Exeter EX4 4QD, UK

Keywords: BAP module, arabidopsis, hypocotyl elongation, Gene Regulatory Network, signal

Coordination of cell proliferation, cell expansion and differentiation underpins plant growth. To maximise reproductive success, growth needs to be fine-tuned in response to endogenous and environmental cues. This developmental plasticity relies on a cellular machinery that integrates diverse signals and coordinates the downstream responses. In arabidopsis, the BAP regulatory module, which includes the BRASSINAZOLE RESISTANT 1, AUXIN RESPONSE FACTOR 6 and PHYTOCHROME INTERACTING FACTOR 4 transcription factors, has been shown to coordinate growth in response to multiple growth-regulating signals. In this review, we provide an integrative view on the BAP module control of cell expansion and discuss whether its function is conserved or diversified, thus providing new

The ability of plants to optimise their genetically-encoded growth by adjusting developmental programs to fluctuating environmental signals define plant phenology and adaptation. In order to grow appropriately, plants must respond accordingly with their developmental stage and their surrounding environment to adjust the growth of their cells. Therefore, the basal cellular processes governing plant growth - mainly cell proliferation and cell expansion (see 
1 Glossary) - must be highly connected to both external and developmental signals. For

2 example, as seeds germinate below ground, they must first develop in the dark prior to reach

3 light and form new organs. To do so, they activate a developmental pathway called skotomorphogenesis, where hypocotyl elongation is greatly promoted [1]. Since no division occurs during hypocotyl elongation [2], skotomorphogenesis is mainly driven through cell expansion regulation. Here cell expansion refers to the growth of a differentiated and nondividing cell that mainly occurs through increase of vacuolar size through water uptake, whereas mitotic growth during cell proliferation depends on cytoplasmic growth and DNA replication [3].

Regulation of cell expansion relies on the coordinated action of growth-promoting transcription factors (TF). BRASSINAZOLE RESISTANT 1 (BZR1) and PHYTOCHROME INTERACTING FACTOR 4 (PIF4)-dependent developmental pathways are independently activated respectively by brassinosteroid (BR) and darkness, whereas BZR1 and PIF4 are able to form a complex which directly targets DNA [4]. In addition, auxin-induced AUXIN RESPONSE FACTOR 6 (ARF6) transcription factor has been shown to interact with both BZR1 and PIF4 [5]. These transcriptional regulators can physically be connected to each other forming a complex system of information integration known as the BRZ1/ARF6/PIF4 (BAP) module [4-6]. It has been hypothesized that BZR1, ARF6 and PIF4 could form a trimeric complex able to bind to DNA targets [6] but the presence of such a complex remains to be demonstrated.

In addition to these physical interactions, studies revealed that BZR1, ARF6, and PIF4 share a significant number of targeted genes, supporting a model where the components of the BAP module synergistically promote cell growth [4-6]. In particular, 42\% of ARF6-targeted genes are also targeted by both BZR1 and PIF4 [5]. The common targets of the BAP module components controlling cell growth are mainly linked to cell wall modifications and auxin responses including EXPANSIN, SMALL AUXIN UP-REGULATED (SAURS) and AUX/IAA genes [5]. PACLOBUTRAZOLE RESISTANT 1 (PRE1) which is an HLH transcription factor that binds and inhibits the growth repressor ILI1 BINDING bHLH PROTEIN1 (IBH1), is also among the common targets of the BAP module thus promoting cell expansion $[5,23]$. Nevertheless, beyond the shared regulation of common targets, individual signalling pathways also act independently to control specific targets [5]. Altogether, the BAP module components act as key activators of growth during skotomorphogenesis and may provide a general 
1 the contours of an intricate network of protein interactions and transcriptional regulation

2 underpinning cell expansion making the BAP module a central regulator of growth in various developmental contexts and in different plant species. Beyond its fundamental role in cell expansion regulation, the BAP module forms a precise signal integration system from which the molecular bases are starting to be unravelled.

Integration of hormonal pathways

The BAP module proteins have either individually or collectively been shown to modulate hormonal signalling during growth and development [5]. For example, the transcriptional regulator BZR1 is dynamically regulated by the BRASSINOSTEROID INSENSITIVE 2 (BIN2) GSK3-like kinase [9]. Phosphorylated BZR1 inactive form is then unable to trigger $\mathrm{BR}$ responses. Besides, BZR1 binds and represses genes involved in BR synthesis, creating a negative feedback loop on BR homeostasis [10]. BZR1 is therefore a central regulator of BR signalling through regulating gene expression that defines wider physiological responses. This involves interaction/coordinated action with other key regulators, including those of the BAP complex. PIF4 was also shown to target key auxin biosynthesis genes to promote hypocotyl elongation [11,12]. Interestingly, it was recently shown that PIFs also directly control BR signalling and responses through regulating key BR synthesis genes such as DWF4 and BR6ox2 [13,14]. Additionally, BZR1 directly targets PIF4 promoter and inhibits its transcription in arabidopsis (Arabidopsis thaliana) creating a new regulatory feedback loop [15] (see below). Thus, the BR and auxin signalling pathways are interconnected through the key BAP module components for a coordinated output. In addition to auxin and BR, Gibberellins (GA) are crucial regulators of plant growth as they control both cell division and cell expansion [16]. The DELLA proteins form a family of growth repressors rapidly degraded in presence of GA $[17,18]$. DELLAs trigger cell growth inhibition by preventing PIF4 and BZR1 binding to their DNA targets $[6,17,18]$. DELLAs also promote proteasomemediated degradation of PIF4, providing an additional layer of regulation [19]. Finally, DELLA proteins bind to ARF6 and trigger the inhibition of the auxin-mediated responses [5].

29 Taken together, those results suggest that GA signalling and DELLA proteins could act as 30 major regulators balancing the activity of BAP module.

31 In addition, other input routes have been identified to regulate BAP module activity. JUNGBRUNNEN1 (JUB1), a NAC family transcription factor, regulates GA, BR and light- 
1 mediated signalling, as it directly represses GA3oxl, DWARF4 and PIF4 genes expression, 2 consequently reducing the synthesis of both GA and BR, as well as decreasing response to changes in light signals [20]. JUB1 directly up-regulates the expression of two DELLAs namely GA INSENSITIVE (GAI) and RGA-LIKE 1 (RGL1) [21]. Finally, PIF4 and BZR1 directly repress JUB1 transcription forming a feedback loop [21]. Consequently, JUB1 stands as a major negative regulator of the BAP module activity.

ETHYLENE RESPONSE FACTOR72 (ERF72), another regulator of the BAP module activity, interacts with BZR1 and ARF6 to inhibit the transcription of BZR1- and ARF6targeted genes, BEE3 and XTH7 [22]. ERF72 partial inhibition of the BAP module was also shown to be light-modulated, as light triggers nuclear re-localisation of ERF72 [22]. Thus, ethylene signalling pathway also seems to converge towards the BAP module to regulate growth, as ERF72 would inhibit the growth promoting BAP module following a shade-tolight transition. The BAP module network might be even more intricate as ERF72 can also interact with DELLA proteins [23]. In the light of the evidences discussed here, it is immediately apparent that the BAP module plays a critical role in coordinating hormone signalling and responses that govern growth (Fig.1).

\section{Coordination of response to exogenous cues}

Responses to light and circadian clock

The ability of plants to reprogram their growth and development in response to exogenous environmental cues underpins adaptation. It has been shown that cell expansion sustaining hypocotyl elongation in the dark is controlled by PIFs [24]. In the light, PIF4 level rapidly decreases through proteasome degradation activated by phyB photoreceptor machinery [17,24,25]. Additionally, BIN2 BR-signalling inhibitor can directly mediate PIF4 destabilization [26]. In the dark, DE-ETIOLATED1 (DET1) and CONSTITUTIVE PHOTOMORPHOGENESIS1 (COP1) signalling machinery negatively regulate ELONGATED HYPOCOTYL 5 (HY5), which inhibits PIF4 activity by targets competition [27-29]. Interestingly, HY5 has also been shown to negatively regulate BZR1 at the protein level [30]. Moreover in the dark, COP1 promotes BZR1 phosphorylated forms proteasome degradation counterbalancing BIN2 inhibition [31]. COP1 was also shown to directly bind BIN2 in the dark, adding another level of BR signalling modulation in response to light variations [32]. Finally, under blue light, the photoreceptor CRYPTOCHROME 1 (CRY1) 
1 can bind to both PIF4 and BZR1 and prevent their DNA-binding activity [33,34]. CRY1 also 2 interacts with BIN2 to promote BZR1 phosphorylation [34]. Taken together, these features 3 are in line with the increasing role of brassinosteroids in photomorphogenesis and light 4 signalling [35].

5 GA metabolism is dramatically remodelled upon light exposure: the light signalling pathway allows the transcriptional regulation of GA metabolism genes expression to lower GA levels [36]. Light thus triggers the BAP module inhibition and stops hypocotyl elongation through DELLA activation. B-Box transcription factors (BBX) are emerging as key coordinators of hormonal pathways and light regulated developmental processes through the BAP module activity [37]. For instance, the BBX24 transcriptional regulator has been shown to physically interact with DELLA in response to shade, reducing its inhibitory activity toward PIF4 and thus promoting shade avoidance response (SAR) [38].

The circadian clock is also an important regulator of the BAP activity controlling notably PIF4 and ARF6 expression [39-41]. The evening complex (EC), composed of EARLY FLOWERING3 (ELF3), ELF4 and LUX ARRHYTHMO (LUX) proteins, binds to the PIF4 promoter at the end of the day and reduces its expression [42]. Meanwhile, an ECindependent PIF4 regulation mediated by ELF3 has been demonstrated [43]. Recently, COP1 SUPPRESSOR 4 (CSU4) has been shown to coordinate CIRCADIAN CLOCK ASSOCIATED1 (CCA1), the main upstream EC regulator, and PIF4 expression [44]. Furthermore, ARF6 has been identified as a direct target of CCA1 [40]. These findings highlight the interplay between circadian clock and BAP-mediated cell expansion.

\section{Responses to temperature}

Temperature is a key environmental signal resulting in adaptive modulation of growth and development. Exaggerated hypocotyl elongation is a characteristic feature of thermomorphogenesis, with PIF4 as the central regulator [45,46]. Indeed, a pif4 loss-offunction mutant is insensitive to high temperatures [45]. At high temperatures, PIF4 repression by ELF3 is released as ELF3 binding to PIF4 promoter is reduced [47]. In addition, phyB is less effective in PIF4 light-mediated degradation increasing PIF4 stability [48]. Interestingly, increased temperatures promote DET1- and COP1-mediated PIF4 gene expression and protein stabilisation [29]. This is further reinforced by the coordinated competitive regulation of PIF4 function by HY5, which inhibits PIF4-mediated induction of target genes at lower temperatures [29]. 
1 Consistent with their cooperative functions in regulating cell elongation, the BAP module 2 proteins play a key role in temperature responsive growth. A positive feed forward loop involving PIF4, auxin, BR and BZR1 that controls temperature responsive growth has been recently identified [15]. PIF4 promotes auxin biosynthesis at elevated temperatures through activating auxin biosynthesis genes [11,12], whereas auxin-dependent cell elongation has been shown to be dependent on BR accumulation and the subsequent activation of BZR1, which in turn promotes PIF4 function through transcriptional activation of PIF4 [15]. This is further supported by the role of PIF4 in BR biosynthesis directly through activating DWF4 and BR6ox2 $[13,14]$. This, together with the cooperative function of PIF4 and BZR1 in activating common target genes for cell elongation $[4,5]$ further strengthens the argument in favour of the BAP module playing a key role in response to temperature.

Responses to various abiotic and biotic stresses

It is becoming increasingly clear that the BAP module plays a key role in optimising growth in response to exogenous cues. Being one of the central growth control module, the BAP module is targeted during various stress conditions for growth repression through the reduction in GA levels and the consequent activation of DELLA proteins that negatively regulate the BAP module output (Fig.2) [49]. For example, salt-treated plants show low GA concentration and high DELLA levels, similarly to plants raised in a cold environment or under osmotic stress [50-55]. A balanced GA/DELLA response thus integrates various sources of abiotic stresses, triggering or inhibiting cell growth. In the particular case of nutrient starvation such as reduced carbon availability, the down regulation of the BAP module occurs through inhibiting TARGET OF RAPAMYCIN (TOR) kinase activity, which is required for $\mathrm{BR}$ responses [56,57]. In response to stress, plants produce reactive oxygen species (ROS) that can damage cells beyond a limit concentration. However, ROS trigger JUB1 activation, which allows a higher tolerance to stress and helps regulating $\mathrm{H}_{2} \mathrm{O}_{2}$ concentration [20]. In addition, DELLA proteins promote survival by maintaining a low level of ROS in cells [58]. Additionally, PIF4 is a molecular link that coordinates growth and immunity in response to seasonal cues $[59,60]$. In nature, plant adaptation to the prevailing environmental conditions is indeed dependent on the coordination of growth and immunity due to the apparent trade-offs

31 between the two processes. In agreement with its synergistic role in growth control with PIF4, 32 BZR1 also plays an important role in balancing the trade-off between growth and immunity 
1 [61]. While both PIF4 and BZR1 indeed promote hypocotyl elongation, they also act to 2 suppress immunity $[29,61]$.

3 Thus, the central role of the BAP module in the environmentally modulated growth further 4 exemplifies its significance as a major regulatory hub in plants (Fig. 1).

\section{Modulation of chromatin state}

All the components of the BAP module have been shown to be active transcription factors, that can directly control target gene expression. Besides the transcriptional regulation of BAP module components, regulation of their downstream targets provides an additional level of control. For instance, BAF60, which encodes a SWI/SNF chromatin remodeler, competes for some PIF4-regulated targets and acts as an antagonist to hypocotyl growth-promoting genes [62]. Moreover, both the light signalling pathway and the circadian clock regulates BAF60 expression [62], providing an epigenetic framework for the integration of environmental signals. Additionally, BZR1 facilitates BR-responsive chromatin remodelling and antagonizes Polycomb repression through the recruitment of a histone 3 lysine 27 (H3K27) demethylase [63]. BZR1 can also mediate chromatin repression via the interaction with the transcriptional co-repressor TOPLESS (TPL) and the subsequent recruitment of histone deacetylases [64]. The possible role of the BAP module in regulating gene expression through chromatin remodelling is further supported by the findings of auxin-mediated transcriptional regulation through differential recruitment of TPL/HDAC co-repressor or SWI/SNF chromatin remodeler $[65,66]$. These evidences suggest chromatin remodelling as a key route through which the BAP module may be orchestrating a wide array of responses in plants to coordinate growth.

\section{The BAP module in various developmental contexts and across species}

With the increasing role of the BAP module in coordinating cell elongation in arabidopsis hypocotyl and young seedlings, it is legitimate to explore a wider role for this module in growth and development, and more generally, in other species. As BR, GA, auxin, temperature and light profoundly affect plant architecture, morphogenesis and senescence, we envisage that the BAP module could constitute a relevant network to drive developmental 
1 processes in the further stages of arabidopsis development and in various environmental 2 conditions as a universal growth-promoting complex.

3 Several lines of evidences favour this hypothesis. First, some BAP module components control the development of other organs. Stamen elongation depends on cell expansion rather than cell proliferation in the epidermis [67] The double arf6 arf8 loss-of-function mutant for auxin responses TFs displays delayed stamen elongation and affects fertilization [68]. ARF8 emerges as an important regulator of stamen filament elongation as one tissue-specific splice variant is able to trigger IAA19 expression allowing late stamen development [69]. Due to its partial redundancy with ARF6 to regulate organ elongation, ARF8 might be an additional member of the BAP module although its molecular interactions with the BAP module TFs remain to be investigated. Functional redundancies have also been shown within the PIF family [70]. These functional redundancies may enhance developmental robustness [71]. Additionally, the dynamic regulation of PIF activity by the spectral quality of light through the photoreceptors underpins SAR and neighbour-detection [72] implying that PIF triggered growth responses are conserved in various developmental contexts.

Second, the BAP module control of cell expansion and its regulatory interactions are also conserved in other species. For instance, down-regulation of SlARF6 and SIARF8 leads to reduced vegetative growth suggesting that their function is conserved in tomato [73]. SIPIF4 expression is regulated by light similarly to AtPIF4 highlighting evolutionarily conserved function of the PIF4 clade [74]. Furthermore, tomato plant over-expressing AtJUB1 display BR- and GA-deficiency morphological phenotypes [75]. Thus JUB1 is a negative regulator of BR, GA and PIF4 in both arabidopsis and tomato. Taken together, these observations highlight the conservation of cell expansion molecular regulators across phylogenetically distant species like tomato and arabidopsis, that diverged approximatively 112 Mya ago [76]. More generally, in maize, ZmPIF4 physically interacts with the arabidopsis DELLA protein RGA [77]. In rice, OsBZR1 is able to promote the expression of INCREASED LEAF INCLINATION1 (ILI1), the PRE1 homolog gene, which in turns targets and inhibits OsIBH1 [78]. Therefore, the components of the BAP module and their downstream effectors seem to also be conserved in monocotyledonous species. This conservation level highlights the fact that the BAP module constitutes a central growth regulator with ancestral functions predating the split between monocotyledonous and dicotyledonous species. 


\section{Concluding remarks}

2 The apparent conservation and pleiotropy of the BAP module suggests that it plays a crucial 3 role during plant evolution allowing developmental plasticity. Major signalling pathways 4 promoting growth through cell expansion regulation converge to the BAP module. The different interactions between its components enable the integration of different environmental and hormonal pathways to provide a coordinated growth output. Ultimately, the functioning of this molecular regulatory hub allows adaptive growth (Fig. 3).

The BAP module activity could impinge upon the development of several arabidopsis organs. However, it is difficult to assess whether these pleiotropic roles of the BAP module members involve cell expansion per se (i.e. growth of an already differentiating and non-dividing cell). Cell proliferation in meristems heavily depends on the coupling between mitotic growth and cell division. By controlling the expression of cell wall loosening enzymes as well as auxin production, members of the BAP module may also play a key role during proliferative development but experimental evidences supporting this idea are still lacking. In addition to their role on growth promotion, BAP members could also have more specific effects depending on where they are expressed. For instance, upon exposure to high temperature, PIF4 accumulates in the future stomatal cells to negatively regulates SPEECHLESS thus preventing stomata differentiation [79].

Phytohormones signalling pathways and environmental responses are conserved within angiosperms. Thus, we suggest that the presence of the BAP module as a central regulatory hub for development control might be shared by a large amount of plant species, involving similar TFs and connecting the same signalling pathways. As a similar molecular framework is involved in different plant organs or within species, we think that the BAP module constitutes a potential lead for crop improvements. Understanding the BAP module pleiotropy within organs could help deciphering the regulation of growth and the genetic basis of pleiotropy (whether it is a cis-regulatory element or a specific amino-acid residue critical for protein-protein interactions) will provide potential targets for new genome editing tools to improve crop performance.

Finally, it is important to notice that cell behaviour is integrated at higher level within organismal growth. Because plant development depends on the coordination of both cell expansion and cell division, there are regulatory connections that link these two processes. Therefore, the BAP module must be seen in a more global picture integrated within a cell that 
1 grows and divides, and that is embedded within a tissue in an organ. This implies additional

2 layers of regulation as one can easily imagine that cell expansion has to be coordinated at 3 higher level notably with mechanical stresses [80]. Mechanical stresses emerge as important 4 drivers for growth and development since they connect to core gene regulatory networks. For instance, SHOOTMERISTEMLESS (STM) expression is modulated through both an auxindependent pathway and an auxin-independent mechanotransduction pathway which provide a synergistic framework for the regulation of meristem identity [81]. This is providing future directions to understand how BAP-mediated growth is integrated at the organ level. Therefore it is a challenging time for plant biologists that calls for the development of quantitative tools and multi-scale analysis to allow an integrative view of biological processes ([82] and Outstanding Questions). A detailed understanding of molecular networks will indeed provide invaluable data. Nevertheless, integrating those networks into interconnected networks and at higher scales will be the only way to understand how growth is continuously optimized to respond to environment.

\section{Acknowledgements}

We thank Véronique Pautot and Patrick Laufs for critical comments. NB is funded by an allocation from École Normale Supérieure de Lyon. NA is supported by the Agence Nationale de la Recherche grant LEAFNET (ANR-12-PDOC-0003). The IJPB benefits from the support of the Labex Saclay Plant Sciences-SPS (ANR-10-LABX-0040-SPS).

1 Fankhauser, C. and Chory, J. (1997) Light Control of Plant Development. Annu. Rev.

2 Gendreau, E. et al. (1997) Cellular basis of hypocotyl growth in Arabidopsis thaliana. Plant Physiol. 114, 295-305

303 Sablowski, R. and Carnier Dornelas, M. (2014) Interplay between cell growth and cell $31 \quad$ cycle in plants. J. Exp. Bot. 65, 2703-2714

324 Oh, E. et al. (2012) Interaction between BZR1 and PIF4 integrates brassinosteroid and 33 environmental responses. Nat. Cell Biol. 14, 802-809

345 Oh, E. et al. (2014) Cell elongation is regulated through a central circuit of interacting transcription factors in the Arabidopsis hypocotyl. Elife 3:e03031 
16 Bai, M.Y. et al. (2012) Brassinosteroid, gibberellin and phytochrome impinge on a 2 common transcription module in Arabidopsis. Nat. Cell Biol. 14, 810-817

37 Singh, A.P. and Savaldi-Goldstein, S. (2015) Growth control: Brassinosteroid activity 4 gets context. J. Exp. Bot. 66, 1123-1132

Bai, M.-Y. et al. (2012) A Triple Helix-Loop-Helix/Basic Helix-Loop-Helix Cascade Controls Cell Elongation Downstream of Multiple Hormonal and Environmental Signaling Pathways in Arabidopsis. Plant Cell 24, 4917-4929

He, J.-X. et al. (2002) The GSK3-like kinase BIN2 phosphorylates and destabilizes BZR1, a positive regulator of the brassinosteroid signaling pathway in Arabidopsis. Proc. Natl. Acad. Sci. 99, 10185-10190

He, J.-X. et al. (2005) BZR1 Is a Transcriptional Repressor with Dual Roles in Brassinosteroid Homeostasis and Growth Responses. Science 307, 1634-1638

Sun, J. et al. (2012) Pif4-mediated activation of yucca8 expression integrates temperature into the auxin pathway in regulating arabidopsis hypocotyl growth. PLoS Genet. 3:e1002594,

Franklin, K.A. et al. (2011) PHYTOCHROME-INTERACTING FACTOR 4 (PIF4) regulates auxin biosynthesis at high temperature. Proc. Natl. Acad. Sci. 108, 2023120235

Wei, Z. et al. (2017) Brassinosteroid Biosynthesis Is Modulated via a Transcription Factor Cascade of COG1, PIF4, and PIF5. Plant Physiol. 174, 1260-1273

Martínez, C. et al. (2018) PIF4 $\square$ induced BR synthesis is critical to diurnal and thermomorphogenic growth. EMBO J. e99552

Ibañez, C. et al. (2018) Brassinosteroids Dominate Hormonal Regulation of Plant Thermomorphogenesis via BZR1. Curr. Biol. 28, 303-310

Sun, T. and Gubler, F. (2004) Molecular Mechanism of Gibberellin Signaling in Plants. Annu. Rev. Plant Biol. 55, 197-223

De Lucas, M. et al. (2008) A molecular framework for light and gibberellin control of cell elongation. Nature 451, 480-484

Feng, S. et al. (2008) Coordinated regulation of Arabidopsis thaliana development by light and gibberellins. Nature 451, 475-479

Li, K. et al. (2016) DELLA-mediated PIF degradation contributes to coordination of

21 Shahnejat-Bushehri, S. et al. (2016) Arabidopsis NAC transcription factor JUB1 light and gibberellin signalling in Arabidopsis. Nat. Commun. 7, 11868

Wu, A. et al. (2012) JUNGBRUNNEN1, a Reactive Oxygen Species-Responsive NAC Transcription Factor, Regulates Longevity in Arabidopsis. Plant Cell Online 24, 482506 regulates GA/BR metabolism and signalling. Nat. Plants 2, 13013

Liu, K. et al. (2018) ERF72 interacts with ARF6 and BZR1 to regulate hypocotyl elongation in Arabidopsis. J. Exp. Bot. 69, 3933-3947 
23 Marin de la Rosa, N. et al. (2014) Large-Scale Identification of Gibberellin-Related Transcription Factors Defines Group VII ETHYLENE RESPONSE FACTORS as Functional DELLA Partners. Plant Physiol. 166, 1022-1032

Leivar, P. and Quail, P.H. (2011) PIFs: Pivotal components in a cellular signaling hub. Trends Plant Sci. 16, 19-28

Leivar, P. and Monte, E. (2014) PIFs: Systems Integrators in Plant Development. Plant Cell 26, 56-78

Bernardo-Garcìa, S. et al. (2014) BR-dependent phosphorylation modulates PIF4 transcriptional activity and shapes diurnal hypocotyl growth. Genes Dev. 28, 16811694

Osterlund, M.T. et al. (2000) Targeted destabilization of HY5 during light-regulated development of Arabidopsis. Nature 405, 462-466

Toledo-Ortiz, G. et al. (2014) The HY5-PIF Regulatory Module Coordinates Light and Temperature Control of Photosynthetic Gene Transcription. PLoS Genet. 10, e1004416

Gangappa, S.N. and Kumar, S.V. (2017) DET1 and HY5 Control PIF4-Mediated Thermosensory Elongation Growth through Distinct Mechanisms. Cell Rep. 18, 344351

30 Li, Q.F. and He, J.X. (2016) BZR1 Interacts with HY5 to Mediate Brassinosteroid- and Light-Regulated Cotyledon Opening in Arabidopsis in Darkness. Mol. Plant 9, 113125

31 Kim, B. et al. (2014) Darkness and gulliver2/phyB mutation decrease the abundance of phosphorylated BZR1 to activate brassinosteroid signaling in Arabidopsis. Plant J. 77, 737-747

32 Ling, J. et al. (2017) Noncanonical role of Arabidopsis COP1 / SPA complex in repressing BIN2-mediated PIF3 phosphorylation and degradation in darkness. Proc. Natl. Acad. Sci. 114, 3539-3544

33 Ma, D. et al. (2016) Cryptochrome 1 interacts with PIF4 to regulate high temperaturemediated hypocotyl elongation in response to blue light. Proc. Natl. Acad. Sci. 113, 224-229

34 He, G. et al. (2019) The Blue Light Receptor CRY1 Interacts with BZR1 and BIN2 to Modulate the Phosphorylation and Nuclear Function of BZR1 in Repressing BR Signaling in Arabidopsis. Mol. Plant DOI: 10.1016/j.molp.2019.02.001

35 Wang, Z.-Y. et al. (2012) Brassinosteroid Signaling Network and Regulation of Photomorphogenesis. Annu. Rev. Genet. 46, 701-724

36 Achard, P. et al. (2007) DELLAs Contribute to Plant Photomorphogenesis. 143, 11631172

37 Vaishak, K.P. et al. (2019) The B-box bridge between light and hormones in plants. $J$. Photochem. Photobiol. B Biol. 191, 164-174

38 Crocco, C.D. et al. (2015) The transcriptional regulator BBX24 impairs DELLA 
activity to promote shade avoidance in Arabidopsis thaliana. Nat. Commun. 6, 6202

Niwa, Y. et al. (2009) The Circadian Clock Regulates the Photoperiodic Response of Hypocotyl Elongation through a Coincidence Mechanism in Arabidopsis thaliana. Plant Cell Physiol. 50, 838-854

Kamioka, M. et al. (2016) Direct Repression of Evening Genes by CIRCADIAN CLOCK-ASSOCIATED1 in the Arabidopsis Circadian Clock. Plant Cell 28, 696-711

Nozue, K. et al. (2007) Rhythmic growth explained by coincidence between internal and external cues. Nature 448, 358-363

Nusinow, D.A. et al. (2012) The ELF4-ELF3-LUX Complex Links the Circadian Clock to Diurnal Control of Hypocotyl Growth. Nature 475, 398-402

Nieto, C. et al. (2015) ELF3-PIF4 Interaction Regulates Plant Growth Independently of the Evening Complex. Curr. Biol. 25, 187-193

44 Zhao, X. et al. (2018) COP1 SUPPRESSOR 4 promotes seedling photomorphogenesis by repressing CCA1 and PIF4 expression in Arabidopsis. Proc. Natl. Acad. Sci. 115, 11631-11636

Koini, M.A. et al. (2009) High Temperature-Mediated Adaptations in Plant Architecture Require the bHLH Transcription Factor PIF4. Curr. Biol. 19, 408-413

Quint, M. et al. (2016) Molecular and genetic control of plant thermomorphogenesis. Nat. Plants 2, 15190

Box, M.S. et al. (2015) ELF3 Controls Thermoresponsive Growth in Arabidopsis. Curr. Biol. 25, 194-199

48 Johansson, H. et al. (2014) Arabidopsis cell expansion is controlled by a photothermal switch. Nat. Commun. 5, 4848

49 Colebrook, E.H. et al. (2014) The role of gibberellin signalling in plant responses to abiotic stress. J. Exp. Biol. 217, 67-75

50 Achard, P. et al. (2006) Integration of Plant Responses to environmentally activated phytohormonal signals. Science 311, 91-94

51 Achard, P. et al. (2008) The Cold-Inducible CBF1 Factor - Dependent Signaling Pathway Modulates the Accumulation of the Growth-Repressing DELLA Proteins via Its Effect on Gibberellin Metabolism. Plant Cell 20, 2117-2129

52 Magome, H. et al. (2008) The DDF1 transcriptional activator upregulates expression of a gibberellin-deactivating gene, GA2ox7, under high-salinity stress in Arabidopsis. Plant J. 56, 613-626

53 Wang, J.-J. and Guo, H.-S. (2015) Cleavage of INDOLE-3-ACETIC ACID INDUCIBLE28 mRNA by MicroRNA847 Upregulates Auxin Signaling to Modulate Cell Proliferation and Lateral Organ Growth in Arabidopsis. Plant Cell 27, 574-590

54 Broeck, L. Van Den et al. (2017) From network to phenotype : the dynamic wiring of an Arabidopsis transcriptional network induced by osmotic stress. Mol. Syst. Biol. 13, 961 
55 Claeys, H. et al. (2012) DELLA Signaling Mediates Stress-Induced Cell Differentiation in Arabidopsis Leaves through Modulation of Anaphase-Promoting. $159,739-747$

Zhang, Z. et al. (2016) TOR Signaling Promotes Accumulation of BZR1 to Balance Growth with Carbon Availability in Arabidopsis. Curr. Biol. 26, 1854-1860

Zhang, Y. et al. (2015) Brassinosteroid is required for sugar promotion of hypocotyl elongation in Arabidopsis in darkness. Planta 242, 881-893

Achard, P. et al. (2008) Plant DELLAs Restrain Growth and Promote Survival of Adversity by Reducing the Levels of Reactive Oxygen Species. Curr. Biol. 18, 656660

Gangappa, S.N. and Kumar, S.V. (2018) DET1 and COP1 Modulate the Coordination of Growth and Immunity in Response to Key Seasonal Signals in Arabidopsis. Cell Rep. 25, 29-37

Gangappa, S.N. et al. (2017) PIF4 Coordinates Thermosensory Growth and Immunity in Arabidopsis. Curr. Biol. 27, 243-249

Lozano-Durán, R. et al. (2013) The transcriptional regulator BZR1 mediates trade-off between plant innate immunity and growth. Elife 2, e00983

Jégu, T. et al. (2017) The Arabidopsis SWI/SNF protein BAF60 mediates seedling growth control by modulating DNA accessibility. Genome Biol. 18, 114

Li, Z. et al. (2018) Brassinosteroid Signaling Recruits Histone 3 Lysine-27 Demethylation Activity to FLOWERING LOCUS C Chromatin to Inhibit the Floral Transition in Arabidopsis. Mol. Plant 11, 1135-1146

Oh, E. et al. (2014) TOPLESS mediates brassinosteroid-induced transcriptional repression through interaction with BZR1. Nat. Commun. 5:4140

Weijers, D. and Wagner, D. (2016) Transcriptional Responses to the Auxin Hormone. Annu. Rev. Plant Biol. 67, 539-574

$66 \mathrm{Wu}$, M. et al. (2015) Auxin-regulated chromatin switch directs acquisition of flower primordium founder fate. Elife 4:e09269

67 Tashiro, S. et al. (2009) Changes in growth kinetics of stamen filaments cause inefficient pollination in massugu2, an auxin insensitive, dominant mutant of Arabidopsis thaliana. Physiol. Plant. 137, 175-187

68 Nagpal, P. et al. (2005) Auxin response factors ARF6 and ARF8 promote jasmonic acid production and flower maturation. Development 132, 4107-4118

69 Ghelli, R. et al. (2018) A Newly Identified Flower-Specific Splice Variant of AUXIN RESPONSE FACTOR8 Regulates Stamen Elongation and Endothecium Lignification in Arabidopsis. Plant Cell 30, 620-637

70 Zhang, Y. et al. (2013) A Quartet of PIF bHLH Factors Provides a Transcriptionally Centered Signaling Hub That Regulates Seedling Morphogenesis through Differential Expression-Patterning of Shared Target Genes in Arabidopsis. PLoS Genet. 9, 
71 Lachowiec, J. et al. (2016) Molecular mechanisms governing differential robustness of development and environmental responses in plants. Ann. Bot. 117, 795-809

Lorrain, S. et al. (2008) Phytochrome-mediated inhibition of shade avoidance involves degradation of growth-promoting bHLH transcription factors. Plant J. 53, 312-323

Liu, N. et al. (2014) Down-regulation of AUXIN RESPONSE FACTORS 6 and 8 by microRNA 167 leads to floral development defects and female sterility in tomato. $J$. Exp. Bot. 65, 2507-2520

74 Rosado, D. et al. (2016) Phytochrome Interacting Factors (PIFs) in Solanum lycopersicum: Diversity, evolutionary history and expression profiling during different developmental processes. PLoS One 11, e0165929

Shahnejat-Bushehri, S. et al. (2017) Arabidopsis NAC Transcription Factor JUNGBRUNNEN1 Exerts Conserved Control Over Gibberellin and Brassinosteroid Metabolism and Signaling Genes in Tomato. Front. Plant Sci. 8, 214

77 Shi, Q. et al. (2018) Functional Characterization of the Maize Phytochrome-Interacting

78 Zhang, L.-Y. et al. (2009) Antagonistic HLH/bHLH Transcription Factors Mediate Brassinosteroid Regulation of Cell Elongation and Plant Development in Rice and Arabidopsis. Plant Cell 21, 3767-3780

$\mathrm{Ku}, \mathrm{H} .-\mathrm{M}$. et al. (2000) Comparing sequenced segments of the tomato and Arabidopsis genomes: Large-scale duplication followed by selective gene loss creates a network of synteny. Proc. Natl. Acad. Sci. 97, 9121-9126

Lau, O.S. et al. (2018) Direct Control of SPEECHLESS by PIF4 in the High-

81 Landrein, B. et al. (2015) Mechanical stress contributes to the expression of the STM Temperature Response of Stomatal Development. Curr. Biol. 28, 1273-1280

Figure Legends

Figure 1. The BAP module is a molecular hub. BZR1, ARF6 and PIF4 interact to allow 
1 and hormonal pathways to provide a coordinated growth output. Various key regulators

2 provide additional levels of complexity to control this gene regulatory network.

Figure 2. Various stresses differentially affect the BAP module activity. Plants use an avoidance strategy in response to starvation, flood or shade events through an activation of the BAP module, as the GA/DELLA balance favors the BAP module activity. In response to salt stress, osmotic stress, cold, the GA/DELLA balance shifts to inactivate the BAP module, promoting stress tolerance. Pathogens lead to BZR1 and PIF4 inhibition. Oxidative stress promotes JUB1 activation. There is an apparent trade-off between BAP-mediated growth promotion and stress tolerance.

Figure 3. Information integration within the BAP module. This integration system senses various signals, process them and provide an integrative output. Due to the complex regulation of its components, the BAP module can receive and process multi-signal informations from both internal (homonal concentrations, circadian clock) and external (light, temperature, stresses...) cues to provide an adapted growth response.

\section{Glossary}

Cell expansion: Biological process allowing organismal growth whereby a cell increases its volume. This process occurs within non-proliferating tissues and relies mainly on an increase of the cell vacuole volume. Cell elongation, as preferential cell growth in one direction, represents a particular case of cell expansion.

Cell proliferation: Biological process allowing organismal growth whereby cells increase in numbers. This process requires the coordination of mitotic cell growth driven by cytoplasmic growth and DNA replication and cell division to give rise to two daughter cells.

27 Developmental Plasticity: Growth adjustment of an organism that does not require genetic 28 changes.

Integration: Mechanism whereby multiple-inputs (often from different natures) are coordinated to give rise to a single optimized output. 
1 Plant growth: Set of biological processes required for a tissue or an organ to increase in size.

2 As no cell migration and little cell death occurs in plants, plant growth is mainly driven by 3 cell proliferation and cell expansion.

4 Plant immunity: Set of biological processes allowing the plant to defend itself against 5 pathogens.

6 Robustness: Biological property of a system to provide an invariable output in response to 7 variable inputs.

8 Skotomorphogenesis: Developmental program in which a plant adjusts its growth to a dark 9 environment. Seedlings form apical hooks and elongated hypocotyls, chlorophyll synthesis is 10 inhibited.

11 Thermomorphogenesis: Developmental program in which a plant adjusts its growth to 12 increased temperature. Seedlings display elongated hypocotyls and petioles. 
Phytohormones

\section{Environment}
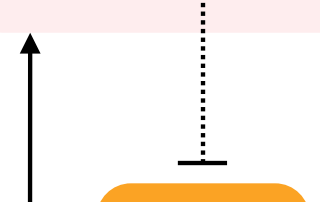

>
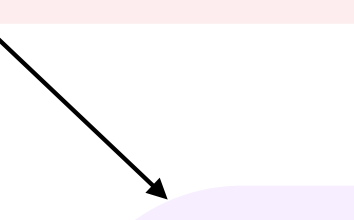

\section{DELLA}

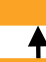

JUB1

ERF72
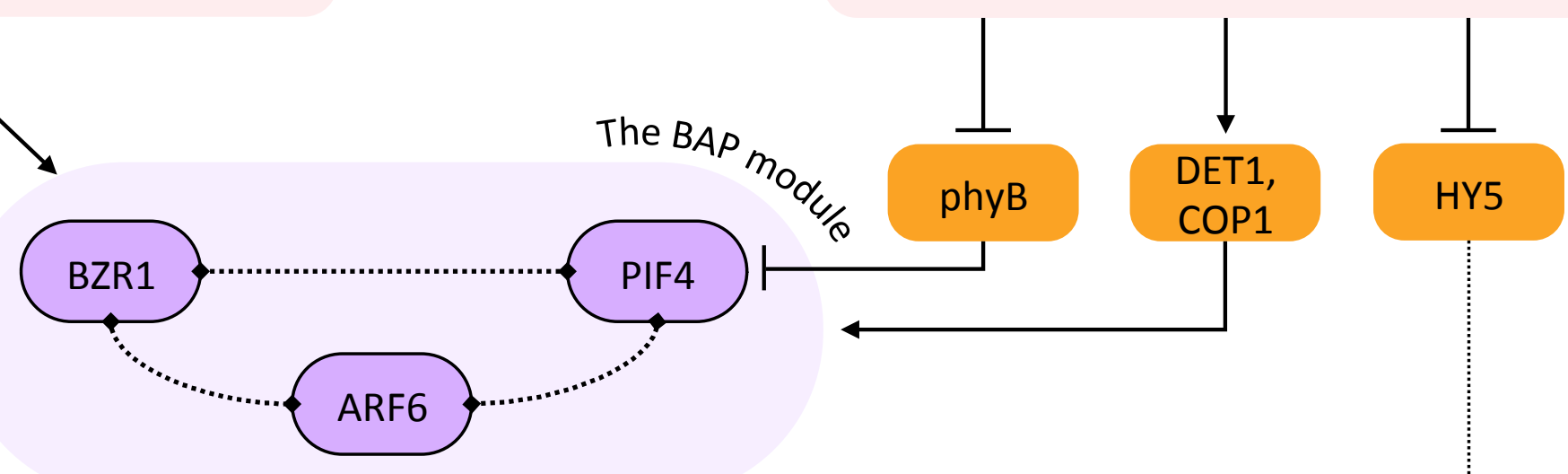

Protein interaction

targets

Transcriptional regulation 


\section{Starvation}

Flood events

Shade

Salt stress

Osmotic Stress

Cold

Pathogens

ROS production
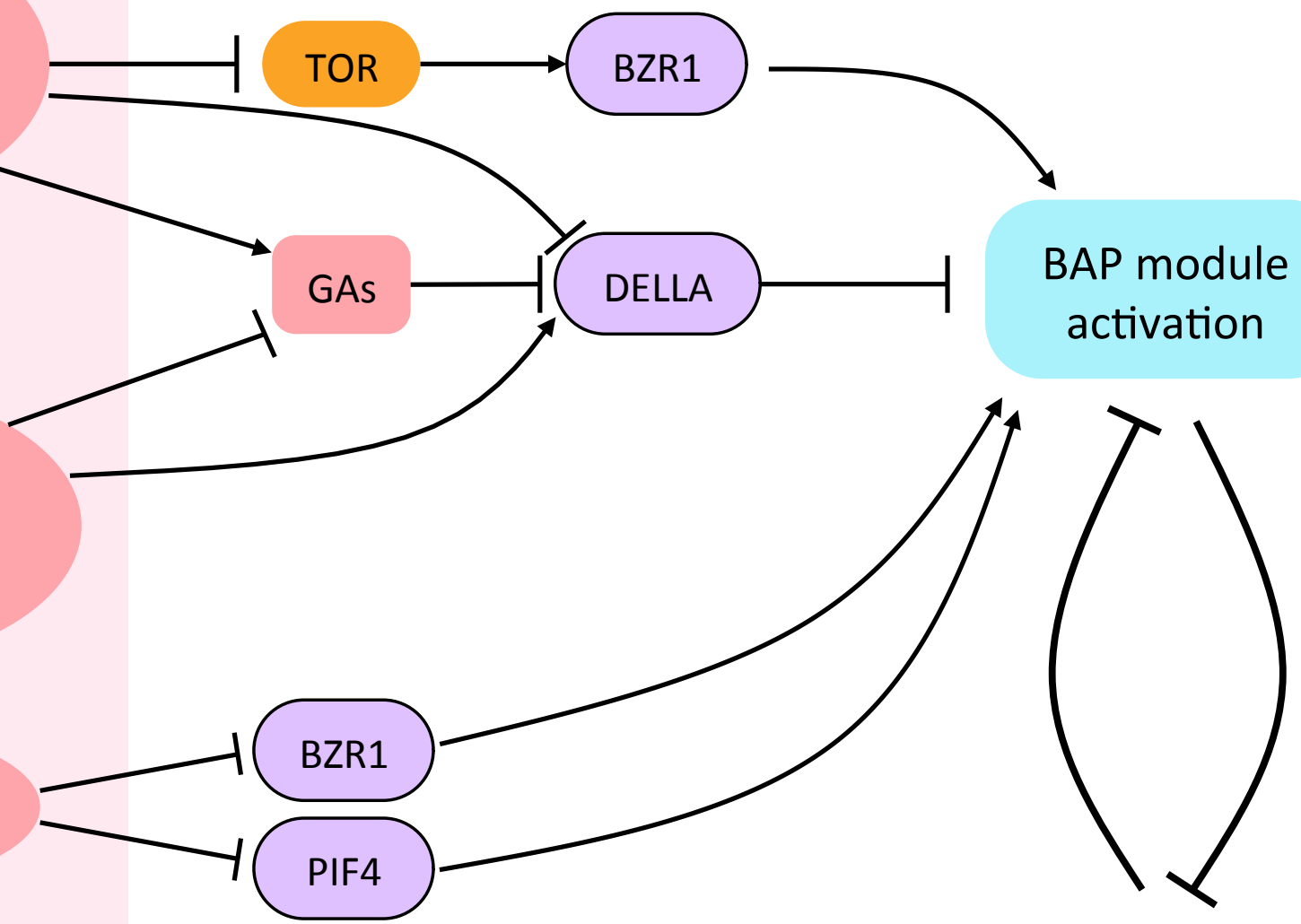

BAP module activation

Stress tolerance Survival Immunity 


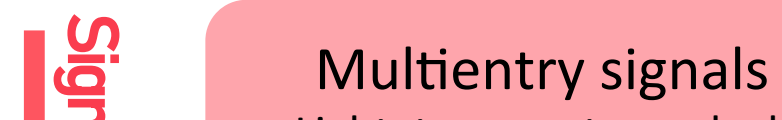

Light, temperature, clock stresses

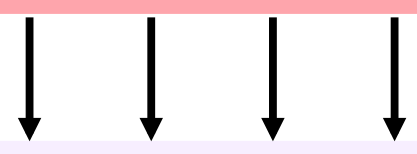

Information integration
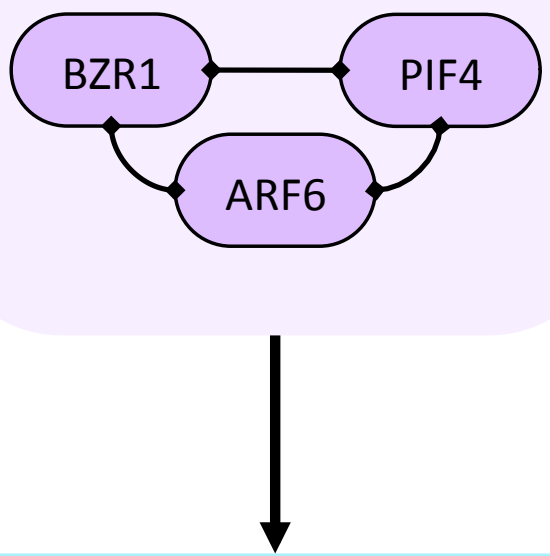

Interdependent \& autonomous effects

Adaptive output 